Article

\title{
Collinear FAST CARS for Chemical Mapping of Gases
}

\author{
Anton Shutov ${ }^{1, *}$, Dmitry Pestov ${ }^{1, \dagger}$, Narangerel Altangerel ${ }^{1}$, Zhenhuan Yi ${ }^{1}$, Xi Wang ${ }^{1}$, \\ Alexei V. Sokolov ${ }^{1,2}$ and Marlan O. Scully 1,2,3 \\ 1 Institute for Quantum Science and Engineering, Texas A\&M University, College Station, TX 77843-4242, \\ USA; dspestov@gmail.com (D.P.); nara.altan@gmail.com (N.A.); yzh@tamu.edu (Z.Y.); \\ xwangphy@gmail.com (X.W.); sokol@physics.tamu.edu (A.V.S.); scully@tamu.edu (M.O.S.) \\ 2 Quantum Optics Laboratory at the Baylor Research and Innovation Collaborative, Baylor University, \\ Waco, TX 76706, USA \\ 3 Department of Mechanical and Aerospace Engineering, Princeton University, Princeton, NJ 08544, USA \\ * Correspondence: tony_shutov@physics.tamu.edu; Tel.: +1-979-204-9103 \\ † Current address: IPG Photonics Corporation, 377 Simarano Dr., Marlborough, MA 01752, USA.
}

Academic Editor: Johannes Kiefer

Received: 12 May 2017; Accepted: 2 July 2017; Published: 8 July 2017

Featured Application: remote spectroscopic mapping of gas emissions and leaks.

\begin{abstract}
We examine the concentration dependence of the Coherent Anti-Stokes Raman Scattering (CARS) signal obtained for gas mixtures at various conditions using the Femtosecond Adaptive Spectroscopic Technique (FAST). We use the CARS signal of the Q-branch vibrational oscillation of molecular oxygen $\left(1556 \mathrm{~cm}^{-1}\right)$ to confirm the quadratic dependence of the coherent signal on the number of molecules in a test volume. In addition, we demonstrate multi-shot FAST CARS imaging of a gas flow in free space by raster-scanning the area of interest.
\end{abstract}

Keywords: optics; coherent anti-Stokes Raman scattering (CARS); Raman scattering; gas imaging; concentration dependence

\section{Introduction}

Both spontaneous and coherent Raman scattering effects have been employed in spectroscopic systems applied to trace detection and identification of chemicals [1,2] and as a method to measure concentrations of various components in gases [3-5], liquids and solids [6,7]. In spontaneous Raman scattering, the signal is directly proportional to the number of molecules interacting with a single beam of input light. By contrast, coherent anti-Stokes Raman scattering (CARS) is a nonlinear process where the molecules are first put into a state of coherent oscillations, resulting in an increased probability for a probe pulse to scatter and produce Stokes or anti-Stokes shifted photons [8]. Therefore, CARS-based techniques possess an inherent ability to offer higher signal strength and faster collection speed compared to approaches based on spontaneous Raman scattering [9].

With the development of ultrashort pulsed laser sources, CARS imaging opened the possibility to study the dynamics of such rapidly changing systems as gas flows [10]. The great flexibility of the CARS technique makes it a popular instrument for performing thermometry measurements for various gases: nitrogen and oxygen [11-13], carbon dioxide [14], as well as methane and hydrogen [15]. CARS techniques allow concentration and temperature measurements in harsh environments and at high temperature during the combustion process [16,17]. Moreover, one-dimensional $[18,19]$, as well as two-dimensional [20] single-shot thermometry and mapping of reactants and products in gas flows can be performed by different experimental approaches. 
The CARS signal is expected to scale proportionally to the second power of the concentration of target molecules in a sample; however, Raman linewidth changes [21], signal re-absorption [7], as well as nonresonant contribution from background molecules [22,23] may alter the dependence of the CARS signal on the concentration, making it difficult to correlate the signal strength with the distribution of those molecules. Gas flows, in particular, are multi-component systems, usually containing a large number of background molecules contributing to the CARS signal background. Some of the aforementioned problems, i.e., Raman line broadening, can be resolved by taking into account line broadening coefficients [24] as well as dephasing rates for the gas species [25].

Here we show that the femtosecond adaptive spectroscopic technique (FAST) for CARS [26] maintains the proper dependence of the CARS signal on concentration and thereby assures a direct correspondence between CARS intensity image and molecular distribution. FAST CARS is a combination of methods aimed at optimizing the CARS signal and suppress the noise background. In the 'hybrid' implementation of FAST CARS, we use two ultrashort pump-Stokes excitation pulses to prepare a coherent oscillation of molecules (Figure 1a), in combination with a narrowband delayed probe pulse to provide near-perfect non-resonant four-wave mixing background suppression $[27,28]$. Recently, in addition to gas-phase studies, this scheme was successfully used for microspectroscopy $[29,30]$, and for spectroscopic molecular sensing aided by plasmonic nanostructures [31-33].
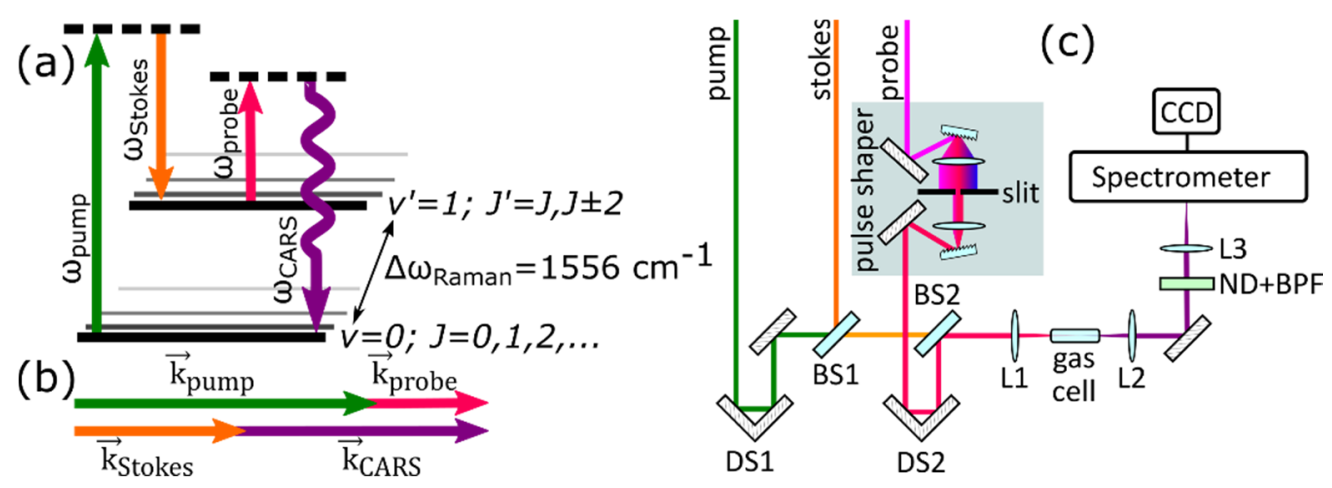

Figure 1. (a) CARS (coherent anti-stokes Raman scattering) level diagram. CARS signal is generated from the probe pulse scattering off the molecular vibration, coherently prepared by the pump $\left(\omega_{\text {pump }}\right)$ and Stokes $\left(\omega_{\text {Stokes }}\right)$ pulses, which are resonant with the Raman frequency of the molecule $\left(\Delta \omega_{\text {Raman }}\right) . v, J, v^{\prime}, J^{\prime}$-initial and final vibrational and rotational states correspondingly; (b) Phase matching CARS scheme for collinear beams configuration; (c) Experimental setup. DS1,2-delay stages. BS1,2-beamsplitters, L1-3-lenses $(f=100 \mathrm{~mm}), \mathrm{ND}+\mathrm{BPF}$ - set of neutral density and bandpass filters.

In this work, we experimentally demonstrate that the hybrid CARS signal obtained for molecular oxygen scales as the square of the number of molecules in two scenarios. We should note that the determination of molecular concentration based on the quadratic dependence of the CARS signal intensity on the number of molecules has been performed before using ns-CARS [34] and hybrid CARS [35] techniques. However, here we focus on demonstrating that this dependence can be maintained at different experimental conditions without prior calibration of specific gases composition or concentration. In addition, we provide a simple yet clear illustration of how the hybrid CARS technique can be exploited for imaging and sensing of a gas flow escaping from a nozzle by performing multi-shot measurements along with a raster-scanning an area in a collinear configuration. In the future, the same ideas can be applied to remote detection and sensing of trace amounts of gases [36]. 


\section{Experimental Setup}

Figure 1c shows the experimental setup for our oxygen concentration measurements. As a laser source, we employ a Ti:sapphire regenerative amplifier $(1 \mathrm{kHz}$ repetition rate, $0.73 \mathrm{~mJ} /$ pulse, Legend, Coherent, Santa Clara, CA, USA) with two equally pumped optical parametric amplifiers (OPAs: OPerA-VIS/UV and OPerA-SFG/UV Coherent, Santa Clara, CA, USA). The outputs of the two OPAs are used as the pump and Stokes pulses $(<130 \mathrm{fs})$. A small fraction of the amplifier output passes through a homemade pulse shaper with an adjustable slit and is used as a probe beam. The adjustable slit width allows us to select a narrow spectral band $\left(\Delta v \approx 11 \mathrm{~cm}^{-1}\right)$ at $806 \mathrm{~nm}$, which corresponds to about 2 ps pulse duration. The pump and the probe beams pass through delay stages (DS1,2), so that the probe pulse is time delayed with respect to the pump and Stokes pulses. Choosing time delay for the probe pulse along with its pulse shaping, provide a clear CARS signal with suppressed non-resonant background from the four-wave mixing signal generated by the three beams.

The collinear geometry configuration for all beams aids to simplify optical alignment and signal collection, with automatically satisfied phase-matching conditions in gases (Figure 1b). The wavelength (FWHM bandwidth) of the pump, Stokes pulses and probe beams are set to $556 \mathrm{~nm}(13.6 \mathrm{~nm}), 610 \mathrm{~nm}$ $(14.1 \mathrm{~nm})$ and $806 \mathrm{~nm}(0.7 \mathrm{~nm})$, with $3.1 \mu \mathrm{J}, 2.6 \mu \mathrm{J}$ and $0.9 \mu \mathrm{J}$ pulse energies respectively. When the probe delay is varied, the full hybrid CARS spectrogram can be observed (for example, see the graphical abstract for this paper). In present experiments, the probe delay with respect to the pump/Stokes pulses is fixed at an optimum values of $2.1 \mathrm{ps}$. The beams are combined by two dichroic beamsplitters (BS1,2) and focused by a convex lens $\mathrm{L} 1(f=100 \mathrm{~mm})$ in the homemade gas cell (Borosilicate glass, $1 \mathrm{~mm}$ thickness, $25.2 \mathrm{~cm}^{3}, 8 \mathrm{~cm}$ long) filled with $\mathrm{N}_{2}$ and air gas mixture. The three beams and the generated CARS signal are collimated by another lens (L2, $f=100 \mathrm{~mm}$ ) and filtered by a set of neutral density (FW2AND Thorlabs, Newton, NJ, USA) and bandpass filters (FF01-732/68 Semrock, Rochester, NY, USA) (ND+BPF). After passing through the filters, the beam is focused by the lens L3 $(f=100 \mathrm{~mm})$ on the entrance slit of the spectrometer: a spectrograph (Chromex Spectrograph 250is, Albuquerque, NM, USA), which has a liquid-nitrogen-cooled CCD camera (CCD: uncoated Spec-10:400B, Princeton Instruments, Trenton, NJ, USA) attached.

The spectral resolution of the experimental setup is limited by two factors: resolution of the spectrometer and the probe pulse spectral width. The spectrometer resolution is determined by its slit size and was set to $0.12 \mathrm{~cm}^{-1}$ for all measurements, thus the total spectral resolution during experiments was mainly limited by the width of the probe pulse $\left(\approx 11 \mathrm{~cm}^{-1}\right)$. The probe pulse duration $(\approx 2 \mathrm{ps})$ constrains the temporal resolution. The spatial resolution in transverse to the beams propagation direction is mainly limited by a beam focal spot diameter (estimated at $\approx 20 \mu \mathrm{m}$ ). However, the axial spatial resolution, i.e., in the direction of the beams propagation, can be potentially limited by the fact that the CARS signal is generated in the region up to 6 times larger than the Rayleigh range $(\approx 0.4 \mathrm{~mm}$ for our beams configuration) [8] in the collinear beams configuration. This fact does not limit the concentration dependence measurements since the gas cell length is significantly larger than the estimated length of this region $(2.4 \mathrm{~mm})$.

\section{Results and Discussion}

In the first set of experiments we study the CARS signal of $\mathrm{O}_{2}$ molecules by filling the gas cell with air at different pressures. As the first step towards optimizing the beam alignment and time delays for pump/probe pulses, we collect the CARS spectrum showing rotational-vibrational structure of oxygen molecule (Figure 2), where several peaks can be clearly distinguished. The signal was collected for $2.2 \mathrm{~s}$ at atmospheric pressure at $1 \mathrm{kHz}$. The main peak of the spectrum $\left(1555.6 \mathrm{~cm}^{-1}\right)$ corresponds to the Q-branch $(\Delta J=0)$ vibrational transition from the vibrational ground level of the oxygen molecule. Smaller peaks on the left and on the right to the Q-branch transition represent O- $(\Delta J=-2)$ and S- $(\Delta J$ $=+2$ ) transitions correspondingly [37]. 


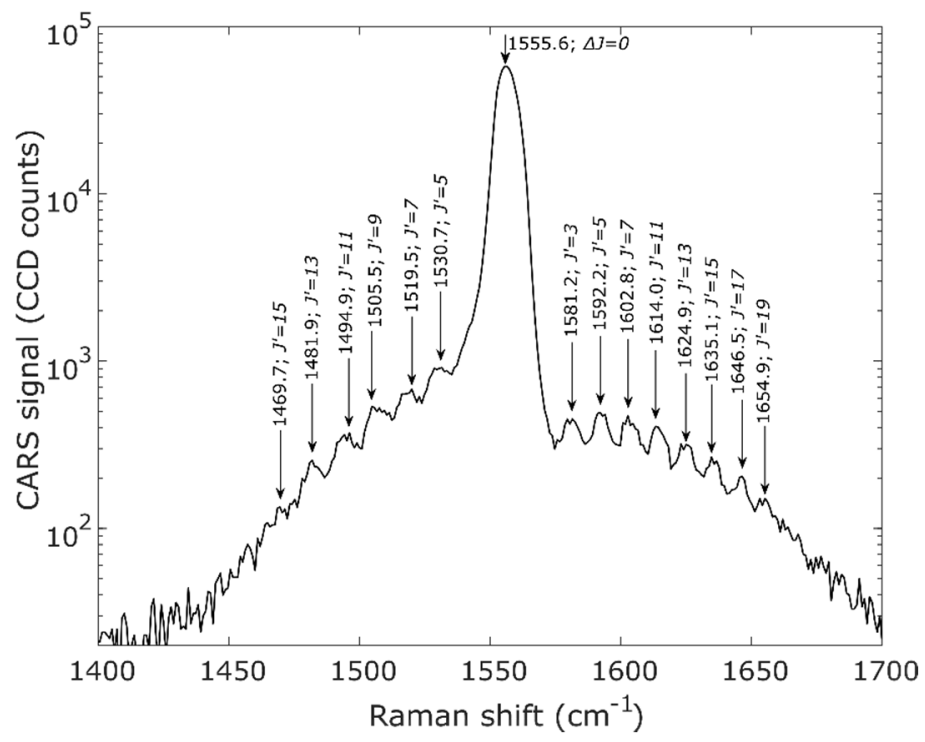

Figure 2. CARS spectrum of molecular oxygen in ambient air. Arrows mark the locations with maximum intensities, and corresponding wavenumbers and final quantum rotational numbers are given.

Thereafter, we use the maximum CCD count value from the region of Raman shift $1556 \pm 1 \mathrm{~cm}^{-1}$, i.e., the intensity of the Q-branch vibrational line to examine the dependence of the CARS signal on gas concentration and pressure. Before calculating the maximum intensity, the zero-pressure background signal was subtracted from the data. We find using the maximum intensity to be more convenient and yet sufficiently precise for studying the dependence of the CARS signal on molecular concentration, when the absolute concentration of gas species is irrelevant. However, it is worth mentioning, that the fitting curves parameters for integrated intensity data points differ from the peak fittings by less than one standard deviation (4.6\%).

During these measurements laser power fluctuations at $1 \mathrm{kHz}$ repetition rate were below $0.5 \%$ and the signal was integrated for $5.2 \mathrm{~s}$. The long integration time aids in significantly reducing the signal intensity fluctuations, but forces us to use at least $\mathrm{ND}=3.0$ filter at ambient conditions in order to avoid saturation of the CCD.

First, the cell is filled with a gas mixture of ambient air and pure nitrogen (Figure 3, solid circles). We assume the oxygen presence in air to be $21 \%$ and vary the partial pressures of air and nitrogen while keeping the total pressure in the cell constant. These measurements allow us to study the dependence of CARS signal on the concentration of $\mathrm{O}_{2}$ with different amounts of background molecules $\left(\mathrm{N}_{2}\right)$. In this case, the CARS signal scales as a square of the relative oxygen concentration.

Next, we fill the cell with ambient air at various pressures, keeping the gas composition and ratio of oxygen molecules to background molecules constant. We control the total pressure inside the gas cell by a ball valve, and perform measurements for the range from 0.01 bar to 1.07 bar. The experimental data with a fit curve are shown in Figure 3 (hollow diamonds), where the signal is proportional to the square of the gas pressure. Hence, we conclude that in both cases of constant gas mixture at different pressures and varied gas compositions at constant pressure, the CARS signal is proportional to the square of the number of $\mathrm{O}_{2}$ molecules. 


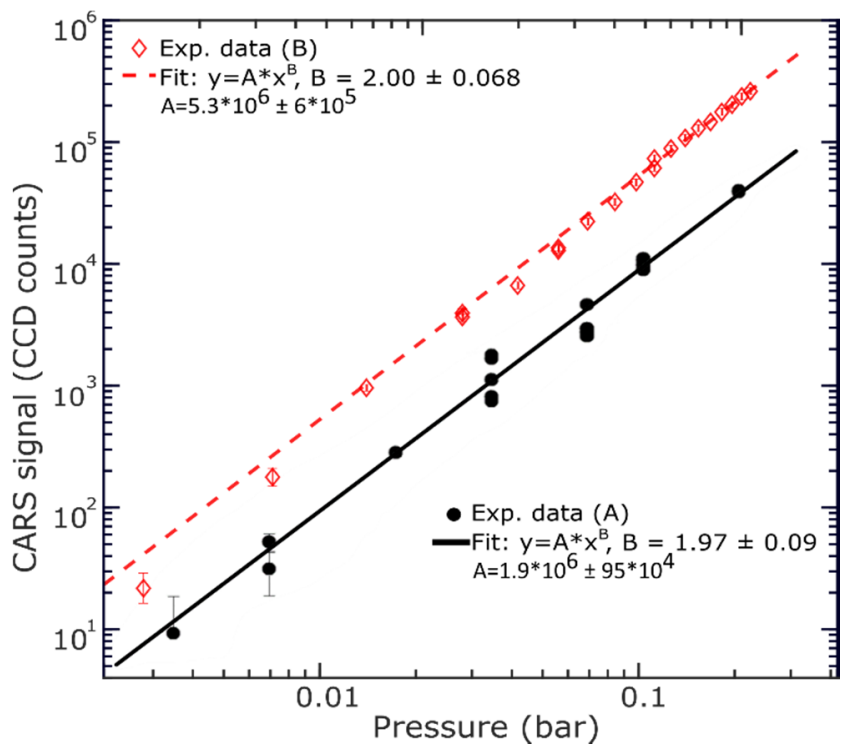

Figure 3. CARS signal dependence on $\mathrm{O}_{2}$ partial pressure at constant total pressure in the cell (black circles, solid line), and at constant gas mixture at different pressures in the cell (red diamonds, dashed line). Both fittings are performed using "power1" fit in MATLAB R2016b (MathWorks, Natick, MA, USA). 95\% confidence bounds are provided for each fitting parameter.

In the next set of experiments, we replace the gas cell with a $1 \mathrm{~mm}$ round nozzle connected to a cylinder with $\mathrm{N}_{2}$ gas and pointed in the direction perpendicular to the beams propagation. Hereafter, we obtain the CARS signal for $\mathrm{O}_{2}$ molecules in ambient air in front of the nozzle in the vicinity of the focal plane of the beam (Figure 4), where zero of the $X$-axis corresponds to the nozzle surface and zero of the $\mathrm{Y}$-axis to the center of the nozzle. After we set the gas pressure such that the gas flow from the nozzle remains constant (estimated at $\approx 4.5 \mathrm{~m} / \mathrm{s}$ ), we move the nozzle in a direction transverse to the axis of the beam propagation direction ( $\mathrm{Y}$ and $\mathrm{X}$ axes in the figure). Hence, by obtaining the CARS signal for $\mathrm{O}_{2}$ at various nozzle positions it becomes possible to visualize the flow of nitrogen from the nozzle. One can see that the nitrogen flow stays almost symmetric about the zero of the Y-axis as it propagates away from the nozzle, where the slight slope can be due to a tilt of the nozzle. The CARS signal from oxygen in the central part of the flow increases very slowly, and the nitrogen diffuses into the surrounding air.

(a)

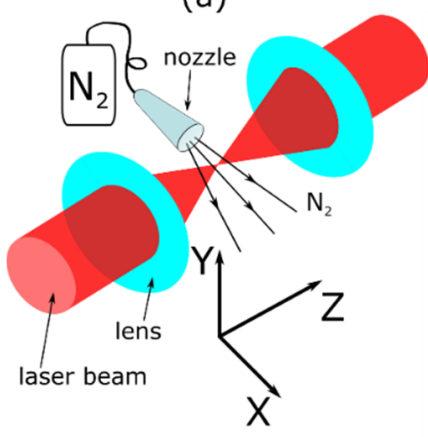

(b)

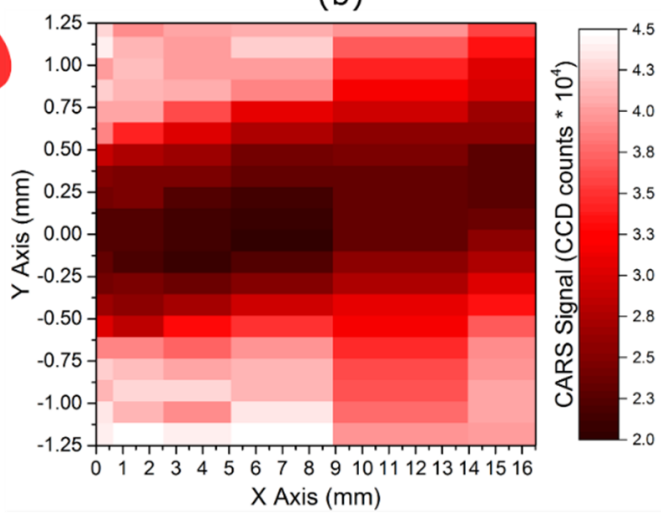

Figure 4. $\mathrm{N}_{2}$ flow as it displaces air: (a) Setup schematics; (b) the CARS signal from $\mathrm{O}_{2}$ in front of the nozzle. Darker regions correspond to higher concentrations of nitrogen. 
We then add a thin metal plate $(50 \times 20 \times 0.5 \mathrm{~mm})$ in front of the nozzle to examine the resulting flow disturbance (Figure 5). The plate is placed at a distance $5.9 \mathrm{~mm}$ away from the nozzle surface in such a manner that the top half of the nozzle is blocked by the plate. The thin plate acts as an impenetrable barrier/obstacle for the gas and laser beams. The flow is disturbed and no longer symmetric; points with zero CARS signal mark the plate location. Moreover, the signal decreases in front of the plate as the nitrogen flow is partially redirected along the surface, while another portion of the flow is deflected by the plate downward. Right behind the barrier, the signal is restored to its value in ambient air since the nitrogen flow cannot penetrate through the plate; i.e., the air in this region stays undisturbed.
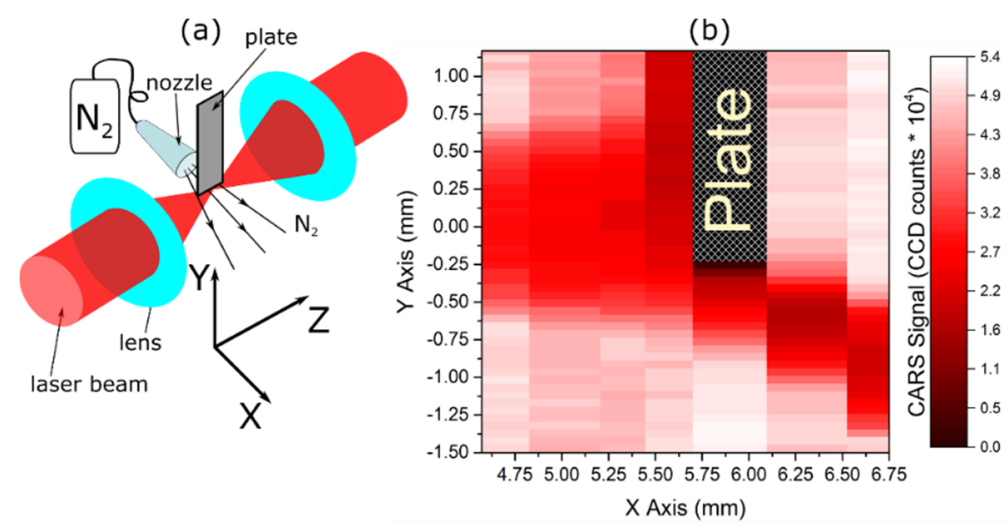

Figure 5. $\mathrm{N}_{2}$ flow as it displaces air with a flat barrier plate placed in front of the nozzle. (a) Setup schematic; (b) $\mathrm{O}_{2}$ CARS signal from air in front of the nozzle. Darker regions correspond to higher concentrations of nitrogen.

\section{Conclusions}

We have experimentally demonstrated the quadratic dependence of the CARS signal of the Q-branch vibrational transition of molecular oxygen on the number of $\mathrm{O}_{2}$ molecules in a gas mixture. We examined two cases. In the first, the mixture was prepared with various amount of background molecules but at constant total pressure. In the second case, the mixture composition remained unchanged while the pressure was varied. No significant difference between these cases was found as both signals showed quadratic dependence on the number of molecules. Furthermore, we have illustrated how CARS can be used for the visualization of gas flow in a simple, free-space configuration, both with a plate barrier and without it. We believe this method is applicable for performing gas flow images for any molecules with any Raman-active modes, as long as the CARS signal can be retrieved with a suppressed non-resonant background.

Acknowledgments: This works is supported by Office of Naval Research (Awards No. N00014-16-1-3054, N00014-16-1-2578), Robert A. Welch Foundation (Grants No. A-1261, A-1547), National Science Foundation (PHY-1307153 and CHE-1609608). Anton Shutov is supported by the Herman F. Heep and Minnie Belle Heep Texas A\&M University Endowed Fund held/administered by the Texas A\&M Foundation. We thank Alexandra Zhdanova and Mariia Shutova for proofreading the manuscript.

Author Contributions: Anton Shutov, Dmitry Pestov and Alexei V. Sokolov designed the experiment and analyzed the data. Anton Shutov, Dmitry Pestov, Narangerel Altangerel, Zhenhuan Yi, Xi Wang performed the experiment. Alexei V. Sokolov, Marlan O. Scully supervised the work. Anton Shutov wrote the manuscript with help of Alexei V. Sokolov, Dmitry Pestov and Zhenhuan Yi.

Conflicts of Interest: The authors declare no conflict of interest.

\section{References}

1. Kneipp, K.; Kneipp, H.; Itzkan, I.; Dasari, R.R.; Feld, M.S. Ultrasensitive Chemical Analysis by Raman Spectroscopy. Chem. Rev. 1999, 99, 2957-2976. [CrossRef] [PubMed] 
2. Dogariu, A.; Goltsov, A.; Pestov, D.; Sokolov, A.V.; Scully, M.O. Real-time detection of bacterial spores using coherent anti-Stokes Raman spectroscopy. J. Appl. Phys. 2008, 103, 036103. [CrossRef]

3. Webber, B.F.; Long, M.B.; Chang, R.K. Two-dimensional average concentration measurements in a jet flow by Raman scattering. Appl. Phys. Lett. 1979, 35, 119-121. [CrossRef]

4. Regnier, P.R.; Moya, F.; Taran, J.P.E. Gas Concentration Measurement by Coherent Raman Anti-Stokes Scattering. AIAA J. 1974, 12, 826-831. [CrossRef]

5. Richardson, D.R.; Lucht, R.P.; Kulatilaka, W.D.; Roy, S.; Gord, J.R. Chirped-probe-pulse femtosecond coherent anti-Stokes Raman scattering concentration measurements. JOSA B 2013, 30, 188-196. [CrossRef]

6. Dogariu, A.; Goltsov, A.; Xia, H.; Scully, M.O. Concentration dependence in coherent Raman scattering. J. Mod. Opt. 2008, 55, 3255-3261. [CrossRef]

7. Zhi, M.; Pestov, D.; Wang, X.; Murawski, R.K.; Rostovtsev, Y.V.; Sariyanni, Z.E.; Sautenkov, V.A.; Kalugin, N.G.; Sokolov, A.V. Concentration dependence of femtosecond coherent anti-Stokes Raman scattering in the presence of strong absorption. JOSA B 2007, 24, 1181-1186. [CrossRef]

8. Nibler, J.W.; Knighten, G.V. Coherent Anti-Stokes Raman Spectroscopy. In Raman Spectroscopy of Gases and Liquids; Weber, P.D.A., Ed.; Topics in Current Physics; Springer: Berlin/Heidelberg, Germany, 1979; pp. 253-299. ISBN 978-3-642-81281-1.

9. Petrov, G.I.; Arora, R.; Yakovlev, V.V.; Wang, X.; Sokolov, A.V.; Scully, M.O. Comparison of coherent and spontaneous Raman microspectroscopies for noninvasive detection of single bacterial endospores. Proc. Natl. Acad. Sci. USA 2007, 104, 7776-7779. [CrossRef] [PubMed]

10. Roy, S.; Gord, J.R.; Patnaik, A.K. Recent advances in coherent anti-Stokes Raman scattering spectroscopy: Fundamental developments and applications in reacting flows. Prog. Energy Combust. Sci. 2010, 36, 280-306. [CrossRef]

11. Roy, S.; Kulatilaka, W.D.; Richardson, D.R.; Lucht, R.P.; Gord, J.R. Gas-phase single-shot thermometry at 1 $\mathrm{kHz}$ using fs-CARS spectroscopy. Opt. Lett. 2009, 34, 3857-3859. [CrossRef] [PubMed]

12. Reichardt, T.A.; Schrader, P.E.; Farrow, R.L. Comparison of gas temperatures measured by coherent anti-Stokes Raman spectroscopy (CARS) of $\mathrm{O}_{2}$ and $\mathrm{N}_{2}$. Appl. Opt. 2001, 40, 741-747. [CrossRef] [PubMed]

13. Matthäus, G.; Demmler, S.; Lebugle, M.; Küster, F.; Limpert, J.; Tünnermann, A.; Nolte, S.; Ackermann, R. Ultra-broadband two beam CARS using femtosecond laser pulses. Vib. Spectrosc. 2016, 85, 128-133. [CrossRef]

14. Kerstan, M.; Makos, I.; Nolte, S.; Tünnermann, A.; Ackermann, R. Two-beam femtosecond coherent anti-Stokes Raman scattering for thermometry on CO2. Appl. Phys. Lett. 2017, 110, 021116. [CrossRef]

15. Dedic, C.E.; Miller, J.D.; Meyer, T.R. Dual-pump vibrational/rotational femtosecond/picosecond coherent anti-Stokes Raman scattering temperature and species measurements. Opt. Lett. 2014, 39, 6608-6611. [CrossRef] [PubMed]

16. Braeuer, A.; Beyrau, F.; Weikl, M.C.; Seeger, T.; Kiefer, J.; Leipertz, A.; Holzwarth, A.; Soika, A. Investigation of the combustion process in an auxiliary heating system using dual-pump CARS. J. Raman Spectrosc. 2006, 37, 633-640. [CrossRef]

17. Tröger, J.W.; Meißner, C.; Seeger, T. High temperature $\mathrm{O}_{2}$ vibrational CARS thermometry applied to a turbulent oxy-fuel combustion process: $\mathrm{O}_{2}$ vibrational CARS thermometry for oxy-fuel combustion process. J. Raman Spectrosc. 2016, 47, 1149-1156. [CrossRef]

18. Kulatilaka, W.D.; Stauffer, H.U.; Gord, J.R.; Roy, S. One-dimensional single-shot thermometry in flames using femtosecond-CARS line imaging. Opt. Lett. 2011, 36, 4182-4184. [CrossRef] [PubMed]

19. Bohlin, A.; Kliewer, C.J. Direct Coherent Raman Temperature Imaging and Wideband Chemical Detection in a Hydrocarbon Flat Flame. J. Phys. Chem. Lett. 2015, 6, 643-649. [CrossRef] [PubMed]

20. Bohlin, A.; Kliewer, C.J. Single-shot hyperspectral coherent Raman planar imaging in the range $0-4200 \mathrm{~cm}^{-1}$. Appl. Phys. Lett. 2014, 105. [CrossRef]

21. Roh, W.B.; Schreiber, P.W. Pressure dependence of integrated CARS power. Appl. Opt. 1978, 17, 1418-1424. [CrossRef] [PubMed]

22. Roy, S.; Meyer, T.R.; Gord, J.R. Time-resolved dynamics of resonant and nonresonant broadband picosecond coherent anti-Stokes Raman scattering signals. Appl. Phys. Lett. 2005, 87, 264103. [CrossRef]

23. Wang, X.; Zhang, A.; Zhi, M.; Sokolov, A.V.; Welch, G.R. Glucose concentration measured by the hybrid coherent anti-Stokes Raman-scattering technique. Phys. Rev. A 2010, 81, 013813. [CrossRef] 
24. Millot, G.; Saint-Loup, R.; Santos, J.; Chaux, R.; Berger, H.; Bonamy, J. Collisional effects in the stimulated Raman $\mathrm{Q}$ branch of $\mathrm{O}_{2}$ and $\mathrm{O}_{2}-\mathrm{N}_{2}$. J. Chem. Phys. 1992, 96, 961-971. [CrossRef]

25. Miller, J.D.; Roy, S.; Gord, J.R.; Meyer, T.R. Communication: Time-domain measurement of high-pressure $\mathrm{N}_{2}$ and $\mathrm{O}_{2}$ self-broadened linewidths using hybrid femtosecond/picosecond coherent anti-Stokes Raman scattering. J. Chem. Phys. 2011, 135, 201104. [CrossRef] [PubMed]

26. Scully, M.O.; Kattawar, G.W.; Lucht, R.P.; Opatrný, T.; Pilloff, H.; Rebane, A.; Sokolov, A.V.; Zubairy, M.S. FAST CARS: Engineering a laser spectroscopic technique for rapid identification of bacterial spores. Proc. Natl. Acad. Sci. USA 2002, 99, 10994-11001. [CrossRef] [PubMed]

27. Prince, B.D.; Chakraborty, A.; Prince, B.M.; Stauffer, H.U. Development of simultaneous frequency- and time-resolved coherent anti-Stokes Raman scattering for ultrafast detection of molecular Raman spectra. J. Chem. Phys. 2006, 125, 44502. [CrossRef] [PubMed]

28. Pestov, D.; Murawski, R.K.; Ariunbold, G.O.; Wang, X.; Zhi, M.; Sokolov, A.V.; Sautenkov, V.A.; Rostovtsev, Y.V.; Dogariu, A.; Huang, Y.; et al. Optimizing the Laser-Pulse Configuration for Coherent Raman Spectroscopy. Science 2007, 316, 265-268. [CrossRef] [PubMed]

29. Shen, Y.; Voronine, D.V.; Sokolov, A.V.; Scully, M.O. A versatile setup using femtosecond adaptive spectroscopic techniques for coherent anti-Stokes Raman scattering. Rev. Sci. Instrum. 2015, 86, 083107. [CrossRef] [PubMed]

30. Shen, Y.; Voronine, D.V.; Sokolov, A.V.; Scully, M.O. Single-beam heterodyne FAST CARS microscopy. Opt. Express 2016, 24, 21652-21662. [CrossRef] [PubMed]

31. Voronine, D.V.; Sinyukov, A.M.; Hua, X.; Wang, K.; Jha, P.K.; Munusamy, E.; Wheeler, S.E.; Welch, G.; Sokolov, A.V.; Scully, M.O. Time-Resolved Surface-Enhanced Coherent Sensing of Nanoscale Molecular Complexes. Sci. Rep. 2012, 2, 891. [CrossRef] [PubMed]

32. Ballmann, C.W.; Cao, B.; Sinyukov, A.M.; Sokolov, A.V.; Voronine, D.V. Dual-tip-enhanced ultrafast CARS nanoscopy. New J. Phys. 2014, 16, 083004. [CrossRef]

33. Hua, X.; Voronine, D.V.; Ballmann, C.W.; Sinyukov, A.M.; Sokolov, A.V.; Scully, M.O. Nature of surface-enhanced coherent Raman scattering. Phys. Rev. A 2014, 89, 043841. [CrossRef]

34. Beyrau, F.; Seeger, T.; Malarski, A.; Leipertz, A. Determination of temperatures and fuel/air ratios in an ethene-air flame by dual-pump CARS. J. Raman Spectrosc. 2003, 34, 946-951. [CrossRef]

35. Engel, S.R.; Miller, J.D.; Dedic, C.E.; Seeger, T.; Leipertz, A.; Meyer, T.R. Hybrid femtosecond/picosecond coherent anti-Stokes Raman scattering for high-speed CH4/N2 measurements in binary gas mixtures: Hybrid fs/ps CARS for high-speed CH4/N2 measurements. J. Raman Spectrosc. 2013, 44, 1336-1343. [CrossRef]

36. Hemmer, P.R.; Miles, R.B.; Polynkin, P.; Siebert, T.; Sokolov, A.V.; Sprangle, P.; Scully, M.O. Standoff spectroscopy via remote generation of a backward-propagating laser beam. Proc. Natl. Acad. Sci. USA 2011, 108, 3130-3134. [CrossRef] [PubMed]

37. Fletcher, W.H.; Rayside, J.S. High resolution vibrational Raman spectrum of oxygen. J. Raman Spectrosc. 1974, 2, 3-14. [CrossRef]

(C) 2017 by the authors. Licensee MDPI, Basel, Switzerland. This article is an open access article distributed under the terms and conditions of the Creative Commons Attribution (CC BY) license (http:/ / creativecommons.org/licenses/by/4.0/). 